\title{
INTRAVENOUS CYCLOPHOSPHAMIDE THERAPY IN ADULTS WITH HENOCH-SCHÖNLEIN PURPURA
}

\author{
doi: $10.1590 / \mathbf{S 1 8 0 7 - 5 9 3 2 2 0 0 8 0 0 0 4 0 0 0 2 5}$
}

Jae Il Shin, Jae Seung Lee - Comment on: Henoch-Schönlein Purpura in adults. Clinics. 2008;63(2):273-6.

We read with interest the article "Henoch-Schönlein Purpura in adults" by López Meiller et al. ${ }^{1}$ They reported 3 adult patients with Henoch-Schönlein nephritis (HSN) (one with hematuria and two with heavy proteinuria) who were treated with IV cyclophosphamide $\left(1 \mathrm{~g} / \mathrm{m}^{2} /\right.$ month for six months). ${ }^{1}$

Although the treatments of severe Henoch-Schönlein nephritis still remains controversial, oral cyclophosphamide has been used in children with severe Henoch-Schönlein nephritis with conflicting results. ${ }^{2-4}$ Tanaka et al. reported that prompt initiation of oral prednisolone $(1.5 \mathrm{mg} /$ $\mathrm{kg} / \mathrm{day}$ ) combined with an 8 -week course of oral cyclophosphamide ( $2 \mathrm{mg} / \mathrm{kg} /$ day) therapy could be effective not only in regressing the renal histologic findings but also in decreasing proteinuria in children with severe Henoch-Schönlein nephritis. ${ }^{2}$ Kawasaki et al. also showed that methylprednisolone and urokinase pulse therapy combined with oral cyclophosphamide was more useful for children with severe Henoch-Schönlein nephritis than methylprednisolone and urokinase pulse therapy alone. ${ }^{3}$ However, when Tarshish et al. performed a randomized controlled study (supportive therapy with or without oral cyclophosphamide $90 \mathrm{mg} / \mathrm{m}^{2} /$ day for 42 days), there were no differences in outcome between the two groups. ${ }^{4}$

Nevertheless, there has been no study on the therapeutic effect of intravenous cyclophosphamide therapy on children or adults with severe Henoch-Schönlein nephritis. On this point, López Meiller et al.'s study ${ }^{1}$ is novel and has important clinical implications for the treatment of severe Henoch-Schönlein nephritis. However, the indications and duration of intravenous cyclophosphamide therapy should also be considered, because this protocol (intravenous cyclophosphamide, $1 \mathrm{~g} / \mathrm{m}^{2} /$ month for six months) which has been previously used in severe diffuse proliferative lupus nephritis can cause various side effects. ${ }^{5}$ Therefore, at least heavy proteinuria or severe histologic findings should be the indications for intravenous cyclophosphamide therapy in severe Henoch-Schönlein nephritis. However it should be noted that one patient in the López Meiller et al.'s study ${ }^{1}$ who showed hematuria without proteinuria was treated with intravenous cyclophosphamide. Also, one or two pulses of IV cyclophosphamide might be enough to control renal inflammations in Henoch-Schönlein nephritis, because two Henoch-Schönlein purpura patients with heavy proteinuria treated with 6 pulses of IV cyclophosphamide had shown a favorable clinical response after only one pulse. ${ }^{1}$

Therefore, further studies should be performed to evaluate the therapeutic effect of IV or oral cyclophosphamide in a large number of adults with severe Henoch-Schönlein nephritis, and the dose and duration of therapy should also be elucidated in the future. 


\section{REFERENCES}

1. López Meiller MJ, Cavallasca JA, Maliandi Mdel R, Nasswetter GG. Henoch-Schönlein Purpura in adults. Clinics. 2008;63:273-76.

2. Tanaka H, Suzuki K, Nakahata T, Ito E, Waga S. Early treatment with oral immunosuppressants in severe proteinuric purpura nephritis. Pediatr Nephrol. 2003;18:347-50.

3. Kawasaki Y, Suzuki J, Suzuki H. Efficacy of methylprednisolone and urokinase pulse therapy combined with or without cyclophosphamide in severe Henoch-Schoenlein nephritis: a clinical and histopathological study. Nephrol Dial Transplant. 2004;19:858-64.
4. Tarshish P, Bernstein J, Edelmann CM Jr. Henoch-Schönlein purpura nephritis: course of disease and efficacy of cyclophosphamide. Pediatr Nephrol. 2004;19:51-6.

5. Omdal R, Husby G, Koldingsnes W. Intravenous and oral cyclophosphamide pulse therapy in rheumatic diseases: side effects and complications. Clin Exp Rheumatol. 1993;11:283-88. 